\title{
O que significa ter uma criança com deficiência mental na família? ${ }^{1}$
}

\section{What does it mean to have a mentally retarded child in the family?}

\author{
Nara Liana Pereira Silva* \\ Maria Auxiliadora Dessen**
}

\begin{abstract}
RESUMO
A família desempenha um papel preponderante no processo de inserção e adaptação da criança deficiente mental no contexto sociocultural. A literatura tem mostrado similaridades e diferenças não só quanto aos modos como as famílias se relacionam com sua criança com deficiência mental, mas também quando comparadas com famílias de crianças com desenvolvimento típico. Este artigo tem como objetivo descrever alguns aspectos do funcionamento de famílias de crianças com deficiência mental, comparando-os àqueles de famílias de crianças com desenvolvimento típico. Ênfase é dada às relações entre os diferentes subsistemas familiares: marido-esposa, genitores-criança e irmão-irmão, e suas implicações para o desenvolvimento infantil. Conhecer a dinâmica das relações de famílias de crianças com deficiência mental constitui o primeiro passo para a compreensão do desenvolvimento e dos mecanismos de adaptação dessas famílias às suas crianças. Mas, para compreender o funcionamento dessas famílias, é preciso levar em consideração as descobertas recentes da genética comportamental.

Palavras-chave: deficiência mental, crianças pré-escolares, família, relações familiares.
\end{abstract}

${ }^{1}$ Este artigo é uma versão modificada da introdução da tese de doutorado da primeira autora, orientada pela segunda. As autoras agradecem o apoio do CNPq.

*Doutora em Psicologia. Universidade de Brasília. liana@unb.br

** Doutora em Psicologia. Professora do Instituto de Psicologia, Universidade de Brasília. dessen@unb.br 


\begin{abstract}
The family plays an important role in the adaptation process of children with mental retardation in the socio-cultural context. Literature has showed us similarities and differences not only in the way that families have been interacting with their mentally retarded child, but also between them and families of typically developing children. So, this article aims to describe some aspects of the family functioning of children with mental retardation comparing them to those from families of children with a typical development. We emphasize the relationships developed in different family subsystems, i.e. husband-wife, parents-child, and child-sibling. In order to understand the family development and adaptation process of children with mental retardation, we need, firstly, to know how these families function. However, to understand the family functioning we need to take into account what we know about behavioural genetics nowadays.

Key-words: mental retardation, preschool children, family, family relations.
\end{abstract}

A deficiência mental tem sido tema de interesse de muitos estudiosos, dentre eles Piaget e Vygotsky, cujas idéias foram muito importantes para a compreensão do desenvolvimento de pessoas com deficiência. Segundo HODAPP, BURACK e ZIGLER (1998), os trabalhos de Piaget trouxeram muitas contribuições para os estudos nesta área, tendo as sequiências do desenvolvimento propostas por ele sido testadas em pessoas DM a partir de 1940, indicando que as crianças DM procediam em seqüência idêntica aos indivíduos com inteligência média. Vygotsky, por sua vez, deu ênfase aos processos que incluíam a linguagem como um mediador do pensamento e aos modos pelos quais os adultos permeiam os comportamentos das crianças. $\mathrm{O}$ foco de sua abordagem é sobre como as crianças se desenvolvem e como o desenvolvimento é alterado quando uma criança tem deficiência mental.

Embora seja inegável a contribuição destes estudiosos, a primeira abordagem evolutiva voltada à deficiência mental foi elaborada somente no final de 1960, por ZIGLER, segundo HODAPP et al. (1998). As principais idéias contidas em sua abordagem incluíam: (a) a hipótese de uma sequiência similar no desenvolvimento, ou seja, as pessoas DM se desenvolveriam lentamente, mas na mesma sequiência que as pessoas com desenvolvimento normal (DN); (b) a existência de uma estrutura de desenvolvimento também similar, ou seja, quando comparadas com pessoas com inteligência média, as pessoas DM deveriam demonstrar as mesmas performances; e (c) os fatores motivacionais e de personalidade desempenhariam um importante papel no desenvolvimen- 
to das pessoas DM. A inclusão deste último aspecto enfatiza não só os processos individuais como os familiares no desenvolvimento da pessoa DM.

Dentre os diversos ambientes de desenvolvimento da criança, a família desempenha um papel preponderante, uma vez que constitui o primeiro ambiente socializador da criança e influencia as relações do indivíduo em outros subsistemas sociais, que extrapolam o âmbito familiar. Dada a importância da família para o desenvolvimento do indivíduo, particularmente de crianças pré-escolares, neste artigo discutimos alguns aspectos do funcionamento das famílias de crianças DM, destacando, na primeira seção, os sentimentos e expectativas de seus membros, bem como as suas reações ao nascimento da criança DM. Na segunda seção, descrevemos a dinâmica das relações entre os diferentes membros que compõem a família dessas crianças, enfatizando o que ocorre nos subsistemas marido-esposa, genitores-criança e irmão-irmão. A terceira seção levanta uma questão importante para reflexão, isto é, se há ou não diferenças nos processos de adaptação de famílias de crianças DM e famílias de crianças com desenvolvimento típico. A última seção tece algumas considerações a respeito de questões atuais que vêm sendo discutidas na Ciência do Desenvolvimento Humano e que devem ser levadas em consideração nos estudos sobre crianças DM.

\section{Como as famílias se sentem tendo uma criança DM?}

O impacto que a criança DM tem sobre a família vem sendo bastante pesquisado nas últimas décadas (BYRNE; CUNNIGHAM, 1985; CRNIC, 1990; FOX et al., 2002; GALLIMORE et al.,1996; KRAUSS; SELTZER, 1998; LEARY; VERTH, 1995; MINNES, 1998; TURNBULL; RUEF, 1996). Embora reconheçamos que este impacto seja significativo para todo o grupo familiar (BRITO; DESSEN, 1999; CASARIN, 1999; KRAUSS; SELTZER, 1998; MINNES, 1998; PEREIRA-SILVA, 2000; PEREIRA-SILVA; DESSEN, 2001; 2002), a literatura tem focalizado sua atenção quase que exclusivamente sobre as mães. Segundo minNEs (1998), os estudos empíricos devotados às experiências de ambos os genitores com sua criança DM não iniciaram antes de 1980. A primeira geração de pesquisas sobre os efeitos da criança com deficiência mental na família apresentava um panorama desanimador, em que o estresse, a sobrecarga, a depressão e o isolamento social eram as características predominantes. Contudo, com a ampliação do número de pes- 
quisas nesta área, os modelos têm passado de interpretações deficitárias de ajustamento para o reconhecimento da possibilidade de uma adaptação mais positiva (SHAPIRO; BLACHER; LOPEZ, 1998).

O estresse é um dos fatores mais comumente investigado em mães de crianças com deficiências (BYRNE; CUNNINGHAM, 1985). Os estudos têm mostrado que mães de crianças DM experienciam mais estresse que os pais e também que as mães de crianças com desenvolvimento normal (DYSON, 1997; HORNBY, 1995; LAMB; BILLINGS, 1997; SHAPIRO et al., 1998; SLOPER et al., 1991), embora DYSON tenha encontrado níveis similares de estresse em mães e pais de crianças DM, em idade escolar. A diferença no nível de estresse encontrado em famílias de crianças com e sem DM pode ser decorrente do fato de que algumas mães de crianças DM teriam mais dificuldades no ajustamento de aspectos pessoais relativos à maternidade (SHAPIRO et al., 1998) e, também, devido aos comportamentos agressivos e ao desempenho de suas crianças (SLOPER et al., 1991). Já as causas do estresse experienciado pelos pais são mais variadas, destacando o temperamento da criança, o isolamento e a retirada social (SHAPIRO et al., 1998), o status empregatício e a satisfação marital (HORNBY, 1995) e, também, a própria avaliação do genitor a respeito do funcionamento da família (DYSON, 1997).

Segundo MINNEs (1998), os estudos sobre estresse na família podem ser agrupados segundo as características da criança deficiente, considerando a combinação de diferentes tipos de deficiências e o impacto da sobrecarga de cuidado com a criança. Estes aspectos são importantes, pois o grau de deficiência da criança tem emergido como um fator que contribui para o estresse parental, particularmente quando associado à dependência da criança, às necessidades de manejo, aos problemas de comportamento e à comunicação limitada. Os genitores têm uma sobrecarga adicional em vários aspectos de sua dinâmica individual e familiar, especialmente no que tange aos aspectos psicológicos, sociais, financeiros, e às atividades de cuidado da criança (ALI et al., 1994; SHAPIRO et al., 1998). Essa sobrecarga, por sua vez, pode estar relacionada aos sentimentos de ansiedade e incerteza quanto às questões de sobrevivência da criança, de seu desenvolvimento, de um compromisso de cuidado prolongado ou, mesmo, do próprio impacto desse cuidado sobre a vida pessoal da mãe, impondo-lhe um senso de limitação e restrição. Os pais de crianças com síndrome de Down também relataram sentir mais sobrecarga que os pais de crianças com desenvolvimento típico (RODRIGUE; MORGAN; GEFFKEN, 1992). A sobrecarga, portanto, pode ser um aspecto desencadeante do estresse e de sentimentos vivenciados pelos genitores de crianças DM. 
Com relação à depressão e ao ajustamento psicológico, alguns estudos mostram que as mães de crianças DM apresentam taxas mais altas de depressão e enfrentam mais problemas com o ajustamento psicológico que os pais (GLIDDEN; FLOYD, 1997; NEGRIN; CRISTANTE, 1996) e que as mães de crianças com desenvolvimento típico (CARR, 1988; SHAPIRO et al., 1998). Mas, no estudo de BRISTOL, GALLAGHER e SCHOPLER (1988), embora um terço das mães de crianças com deficiências fossem consideradas de risco para depressão, os escores não foram significativamente diferenciados para famílias com e sem criança DM. No que tange aos fatores associados à neurose, os estudos apontam para uma não diferenciação entre mães e pais de crianças DM, embora este grupo de genitores apresente maior instabilidade emocional que aquele de crianças com desenvolvimento típico (ALI et al., 1994; SLOPER et al., 1991). Outros sentimentos e estados emocionais podem ser observados nos genitores, tais como mágoa, sofrimento e um contínuo processo de luto (LEARY; VERTH, 1995), além de culpa (ALI et al., 1994).

Embora haja necessidade de se corrigir uma visão unicamente patológica a respeito das reações dos genitores à sua criança $\mathrm{DM}$, os resultados das pesquisas mostram diferenças significativas, pelo menos em algumas dessas dimensões, particularmente entre mães de crianças com e sem DM e, também, entre mães e pais de crianças DM. As evidências sugerem que as mães continuam sendo mais fortemente afetadas pelas obrigações de cuidados com a criança DM. Por outro lado, não se tem clareza se os altos níveis de sintomatologia emocional e física estão relacionados à presença dessa criança ou à rede de variáveis mediadoras ou, ainda, às diferenças culturais entre homens e mulheres (SHAPIRO et al., 1998). Na verdade, todos esses fatores parecem influenciar a natureza e a intensidade das reações à criança DM. Além disso, segundo BYRNE e CUNNINGHAM (1985), os genitores utilizam as mais variadas estratégias para enfrentar os eventos estressantes. Tais estratégias dependem das variações existentes nos estilos cultural e ideológico das famílias e no contexto ecológico em que elas estão inseridas.

Sob o ponto de vista dos irmãos, os estudos indicam que não há dados significativos que apóiem a idéia de um relacionamento problemático entre os irmãos de crianças DM ou que comprovem alguma desvantagem decorrente do fato de se ter um irmão DM (CARR, 1988; ROEYERS; MYCKE, 1995). No estudo de BAGENHOLM e GILLBERG (1991), 65\% dos irmãos de crianças DM e $55 \%$ dos irmãos de autistas percebiam que seu irmão era diferente, porém não tinham palavras para explicar o porquê dessa diferença. Apesar disso, os irmãos de crianças DM brincaram mais com seu irmão DM que os irmãos de crianças autistas e com desenvolvimento típico com seus respectivos irmãos. 
Por outro lado, os irmãos de crianças DM relataram mais sentimentos de insatisfação, por não receberem atenção suficiente dos genitores, embora não tenham relatado problemas de solidão, como os irmãos de crianças autistas. ROEYERS e MYCKE (1995) concluíram que não há diferenças significativas entre os três grupos de irmãos (autistas, DM e DN), no que se refere a estressores específicos e que crianças com um irmão autista ou DM aceitam melhor o seu irmão ou irmã que os irmãos no grupo de crianças com desenvolvimento típico.

Em síntese, ter uma criança DM na família pode ser um evento estressante ou não, trazendo conseqüências para todos os membros em maior ou menor dimensão. Os dados sobre reações, sentimentos, estados emocionais e estresse experienciados por mães, pais e irmãos de crianças DM não são, ainda, conclusivos, requerendo mais investigações para que se possa ter maior clareza sobre o que significa ter uma criança DM na família. Muitas são as variáveis que podem se associar para produzir ou intensificar os sentimentos e estados emocionais dentro da família. Estes sentimentos e emoções têm grande influência no tipo de relacionamento desenvolvido entre a criança e os membros familiares que, por sua vez, têm implicações diretas para o desenvolvimento da criança DM. O tópico a seguir apresenta uma descrição sucinta sobre o que se conhece a respeito das relações desenvolvidas em famílias de crianças DM.

\section{Como é a dinâmica das relações em famílias de crianças DM?}

Os relacionamentos familiares formam um sistema complexo de trocas interativas entre os subsistemas marital e parental que incluem díades, tríades, tétrades e outras constelações, organizadas em um todo integrado, caracterizado por um processo de influências mútuas. Assim, as interações e relações ocorridas no subsistema marido-esposa influenciam aquelas ocorridas em outros subsistemas, especialmente no de genitores-criança (BRISTOL et al., 1988; COWAN; COWAN, 1992; DESSEN, 1997; EMERY; FINCHAM; CUMMINGS, 1992; EREL; BURMAN, 1995; FLOYD; ZMICH, 1991; KERIG; COWAN; COWAN, 1993; KREPPNER, 2000; LAMB; BILLINGS, 1997). 


\section{O subsistema marido-esposa e as relações parentais}

A qualidade da relação marital é um importante determinante das experiências de maternidade/paternidade em todas as famílias, especialmente naquelas com crianças com deficiência mental. Nestas, os genitores têm demandas adicionais com a criação de sua criança DM, geradas pela sobrecarga e estresse devido ao constante cuidado e atenção exigidos pela criança, conforme discutido na seção anterior. No entanto, mães de crianças DM não têm necessariamente mais disfunções em suas vidas que mães de crianças com desenvolvimento típico e, nem sempre, elas têm os piores casamentos ou sistemas sociais de apoio menos adequados (sHAPIRo et al., 1998).

No estudo de FLOUD e ZMICH (1991), com 38 famílias de crianças DM e 34 de crianças com desenvolvimento típico, compostas por mãe, pai e filhos em idade escolar, os resultados indicaram que a insatisfação marital estava mais associada aos problemas de comportamento da criança que à interação marital propriamente dita. As dificuldades de manejo da criança pelos genitores também foram atribuídas mais às limitações cognitivas e à pobre habilidade social da criança que à própria competência dos genitores como pais e mães. O estresse marital, por sua vez, foi associado às demandas de cuidado com as necessidades especiais da criança, às interações maritais negativas e à ocorrência de trocas aversivas com a criança. Um outro estudo com genitores de crianças com e sem DM, sobre o cuidado compartilhado e a satisfação marital, mostrou que a maior participação e envolvimento do pai no cuidado com a criança estavam associados a uma maior satisfação marital (WILOUGHBY; GLIDDEN, apud SHAPIRO et al., 1998).

A literatura relativa aos efeitos da relação marital sobre as relações parentais precisa ser considerada com cautela, pois outros estudos mostram não haver uma diferença entre famílias de crianças com e sem DM quanto ao seu funcionamento, adaptabilidade, coesão e satisfação marital (BRISTOL et al., 1988; SHAPIRO et al., 1998). Shapiro e seus colaboradores também encontraram estudos com um alto índice de divórcio e discórdia marital entre os genitores de crianças com síndrome de Down e outros que não mostraram diferenças entre esses índices quando famílias de crianças com e sem DM foram comparadas. Entretanto, é preciso destacar que há uma variabilidade enorme de mecanismos de adaptação individual utilizados pelas famílias, baseados, em parte, nas características pessoais da criança, na gravidade da deficiência e na competência e recursos dos genitores como cuidadores (BYRNE; CUNNINGHAM, 1985). 


\section{Os subsistemas genitores-filhos e seus estilos de interação}

As interações da criança com ambos os pais influenciam significativamente o desenvolvimento infantil, embora a ênfase da pesquisa recaia no subsistema mãe-criança, que tem sido amplamente investigado nas últimas décadas (BOLSANELLO, 1998; CIELINSKI at al., 1995; COLNAGO, 1991; CORTER et al., 1992; LANDRY et al., 1994; LIEBERMAN; PADAN-BELKIN; HAREL, 1995; MARFO; DEDRICK; BARBOUR, 1998; ROACH et al., 1998; vAUGHN et al., 1994). Apesar da existência de algumas divergências nos resultados das pesquisas sobre a díade mãe-criança DM, há dados consistentes no que tange, principalmente, a uma maior diretividade das mães de crianças DM em relação às mães de crianças com desenvolvimento típico (CIELINSKI et al., 1995; LANDRY et al., 1994; LIEBERMAN et al., 1998; MARFO et al., 1998; SIGOLO, 2000).

Em geral, as interações diádicas mãe-criança são iniciadas mais freqüentemente pelas mães que pelas crianças DM (LANDRY et al., 1994; PEREIRA-SILVA; DESSEN, 2003; SIGOLO, 2000); no entanto, à medida que o bebê cresce, este passa a tomar iniciativa na realização de atividades, especialmente no que tange ao banho e à alimentação (COLNAGO, 1991). PEREIRA-SILVA e DESSEN (2003), ao analisarem a qualidade das interações em seis famílias de crianças com síndrome de Down, verificaram que as interações mãe-criança eram mais amistosas (96\%) que conflituosas, e mais sincrônicas $(76,7 \%)$ que sem sincronia.

As diferenças nas interações mãe-criança com e sem DM são, algumas vezes, relacionadas ao sexo da criança (CIELINSKI et al., 1995; ROACH et al., 1998). Por exemplo, CIELINSKI et al., comparando mães de crianças com desenvolvimento típico e mães de crianças SD, encontraram os seguintes resultados: (a) as mães de meninas SD foram mais diretivas que mães de meninas com desenvolvimento típico; (b) as mães de meninos com desenvolvimento típico foram mais diretivas que as mães de meninas deste mesmo grupo, sendo o padrão inverso observado em crianças SD; (c) meninos com desenvolvimento típico e meninas SD tiveram escores de participação mais altos que meninas com desenvolvimento típico e meninos SD.

Quanto aos pais de crianças DM, eles apresentam comportamentos de comunicação significativamente menos positivos e altas taxas de reciprocidade negativa em comparação com pais de crianças com desenvolvimento típico e com mães de crianças DM (FLOYD; COSTIGAN; PHILLIPPE, 1997; FLOYD; ZMICH, 1991). Já os resultados do estudo de PEREIRA-SILVA e DESSEN (2003), realizado no contexto brasileiro, apontam na direção de pais participativos, 
que iniciam mais interações que as mães, sugerindo possíveis diferenças culturais que precisam ser investigadas mais profundamente.

RODRIGUE et al. (1992), investigando a adaptação psicossocial de pais de crianças autistas, com síndrome de Down e com desenvolvimento típico, não encontraram diferenças significativas entre os três grupos quanto aos cuidados com os filhos e a satisfação percebida por estes. Os pais de meninos relataram maior satisfação com a paternidade que os pais de meninas. No entanto, os resultados do estudo de HORNBY (1995) não mostram contrastes entre a adaptação dos pais às filhas e aos filhos SD. Neste estudo, a adaptação do pai estava relacionada à sua satisfação com o apoio social recebido, à sua satisfação marital e às suas características de personalidade, mais do que às características de sua criança deficiente. Portanto, a literatura mostra dados contraditórios também a esse respeito.

O papel do pai tem sido, recentemente, apontado como uma das tendências de pesquisa sobre as relações familiares (LEWIS; DESSEN, 1999). Esse mesmo panorama caracteriza a literatura sobre deficiência mental, mostrando uma escassez de estudos sobre as interações pai-criança (DESSEN; PEREIRA-SILVA, 2000), que são fundamentais para a compreensão da dinâmica das relações familiares. Os dados atuais refletem a inconsistência existente na área, sugerindo a necessidade de mais pesquisas que incluam não somente o pai em seus planejamentos, mas outros componentes da família, particularmente os irmãos. Mas o que se sabe sobre o relacionamento entre a criança DM e seus irmãos?

\section{O subsistema criança DM-irmão: fatores determinantes e qualidade do relacionamento}

A qualidade do relacionamento entre os irmãos influencia e é influenciada não só pelas relações que os genitores mantêm como marido-esposa, mas também pelas relações que cada um mantém com os filhos, em suas funções de pai e mãe. Por exemplo, a qualidade afetiva do relacionamento de cada genitor com cada uma de suas crianças influencia o relacionamento entre os irmãos, especialmente no tocante à proximidade e amizade entre estes (BRODY; Stoneman, 1996). Para esses autores, os relacionamentos positivos estabelecidos entre os genitores e a criança favorecem o desenvolvimento de com- 
portamentos pró-sociais entre os irmãos; por outro lado, um relacionamento genitores-criança negativo pode conduzir a um relacionamento agressivo entre os irmãos.

Segundo BRody e STONEMAN (1996), o tratamento diferencial dispensado pelos genitores aos filhos, as características individuais dos genitores e das crianças, o relacionamento marital e o clima emocional da família são aspectos que contribuem para as variações no relacionamento entre os irmãos. A literatura (EREL; MARGOLIN; JOHN, 1998) tem apontado uma associação entre a qualidade das relações maritais e das relações entre irmãos, sendo o conflito marital um evento aversivo e causador de sofrimento nos filhos. A infelicidade marital, o conflito e a baixa coesão emocional na família estão associados a um relacionamento caracterizado pela negatividade entre os irmãos (BRODY et al., apud BRODY; STONEMAN, 1996).

O temperamento das crianças é considerado um fator de risco para relacionamentos conflituosos entre os irmãos. No entanto, essa determinação dependerá do contexto de criação da criança, pois há uma associação entre o temperamento da criança e as características do contexto familiar. Crianças com temperamentos difíceis colocam em risco o relacionamento com seu irmão, mas os genitores podem amenizar este risco na medida em que estabelecem e desenvolvem relacionamentos genitores-criança mais positivos. Esta perspectiva amplia o nosso conhecimento e reforça a noção de interdependência entre os vários sistemas familiares, que deve ser considerada na análise da dinâmica e do funcionamento familiar.

Nos últimos anos, houve um aumento no número de pesquisas sobre as relações entre crianças DM e seus irmãos (DESSEN; PEREIRA-SILVA, 2000). STONEMAN (1998) destaca a importância deste tipo de pesquisa, enfatizando a necessidade de dividir os grupos de crianças DM segundo a etiologia: orgânica ou não orgânica. Este cuidado é importante na medida em que as demandas requeridas para cada família podem ser diferentes. Segundo esta autora, os pesquisadores nesta área têm focalizado os irmãos e irmãs de crianças DM com causa orgânica, investigando, principalmente, os seguintes assuntos: o cuidado com os irmãos, a qualidade do relacionamento, o tratamento diferencial e os resultados psicossociais.

É importante compreender os fatores relacionados às diferentes etiologias da deficiência mental que têm relevância para o estudo do relacionamento entre irmãos. Dentre estes fatores, destacam-se as características individuais, como temperamento/personalidade, a competência, as deficiências secundárias e os problemas de saúde (STONEMAN, 1998). Os diferentes tipos de deficiência mental acarretam características comportamentais específicas, tendo um reflexo 
direto no relacionamento entre os irmãos. Por exemplo, crianças com síndrome de Down são consideradas, por alguns, como tendo um temperamento fácil (GANIBAN; WAGNER; CICCHETTI, 1990), enquanto outros consideram uma maior variabilidade no temperamento dessas crianças (GUNN; BERRY; ANDREWS, apud StONEman, 1998). No entanto, os resultados de pesquisas indicam que, afetivamente, essas crianças e seus irmãos têm um relacionamento similar àquele entre irmãos com desenvolvimento típico (ABRAMOVITCH et al., 1987).

No estudo conduzido por CORTER e seus colaboradores, as interações entre a criança com síndrome de Down e seu irmão foram caracterizadas pelo comportamento negativo da criança com síndrome de Down; no entanto, esse resultado foi similar aos encontrados em famílias de criança com desenvolvimento típico. Em comparação com crianças autistas, as crianças SD emitiram mais comportamentos de iniciativa, mas seus irmãos apresentaram uma variedade maior de comportamentos. Contudo, o padrão global de interação mostrado por essas díades foi semelhante ao encontrado em outros estudos com uma amostra diversa de crianças (KNOTT; LEWIS; wILlIAMS, 1995).

A literatura é consistente com relação à assimetria dos papéis entre os irmãos, com o irmão assumindo o papel da criança 'mais velha' e a criança com síndrome de Down assumindo o papel da 'mais jovem', independentemente das idades cronológicas de ambos (ABRAMOVITCH et al., 1987; BRODY et al., 1991; CORTER et al., 1992; KNOTT et al., 1995; STONEMAN et al., 1991). A maioria dos estudos indica, portanto, que a interação se desenvolve com a liderança do irmão no relacionamento, sendo este o responsável pela maior parte das iniciativas de interação, as quais são marcadas por comportamentos pró-sociais e agonísticos. PEREIRA-SILVA (2003) também observou, em sua pesquisa, que os irmãos de crianças com síndrome de Down exerceram mais freqüentemente o papel de 'Dirigente', e a criança SD, o papel de 'Submisso', enquanto, nas famílias de crianças com desenvolvimento típico, ambos os irmãos assumiram o papel de 'Dirigente' em proporções similares.

As diferenças relacionadas ao gênero da criança indicam que irmãs mais jovens de crianças DM fornecem mais ajuda e têm mais responsabilidade com os cuidados diários dispensados aos seus irmãos DM que as irmãs de crianças com desenvolvimento típico (BRODY et al., 1991; STONEMAN et al., 1991). O fato de os irmãos/irmãs de crianças DM ajudarem mais que os irmãos/irmãs de crianças com desenvolvimento típico sugere que a presença de uma criança DM mais velha na família é um poderoso estímulo de cuidado que influencia o comportamento de irmãos e irmãs mais jovens, de uma maneira similar. De certa forma, isto pode revelar que os genitores colocam demandas similares sobre irmãos e irmãs jovens de crianças DM. 
A assimetria de papéis entre os irmãos pode ser conseqüência das limitações na competência da criança DM, o que possibilita aos irmãos com desenvolvimento típico assumirem os papéis de dominantes e controladores. A competência é, portanto, um fator diretamente relacionado à etiologia da deficiência mental. Assim, quanto menos competente for a criança, maior a possibilidade de haver um decréscimo na qualidade da relação entre os irmãos. Os efeitos da criança DM sobre seus irmãos com desenvolvimento típico podem ter vários fatores explicativos; dentre eles, as atitudes e expectativas dos genitores, o tamanho da família, os recursos familiares, a religião, a gravidade da deficiência da criança e o padrão de interação entre os irmãos (BAGENHOLM; GILLBERG, 1991).

Em síntese, as relações desenvolvidas entre os diversos membros da família com a criança DM apresentam tanto similaridades como diferenças quando comparadas às famílias de crianças com desenvolvimento típico. Mas os processos de adaptação destes dois tipos de família às suas crianças são diferentes? A seguir, discorre-se sobre os modos como as famílias se adaptam às suas crianças com e sem DM.

\section{Famílias de crianças com e sem DM: há diferenças nos seus processos de Adaptação?}

Embora um corpo crescente de dados mostre poucas diferenças no processo de adaptação de mães de crianças com e sem DM, usualmente as diferenças encontradas favorecem as mães de crianças com desenvolvimento típico (CIELINSKI et al., 1995; LIEBERMAN et al., 1995; MARFO et al., 1998). Entretanto, isto não significa que as famílias de crianças DM sejam disfuncionais ou pobremente adaptadas, mas, simplesmente, que elas enfrentam mais desafios no processo de educar sua criança DM (sHAPIRo et al., 1998).

As famílias de crianças DM em idade pré-escolar parecem enfrentar as mesmas demandas de famílias que possuem crianças pequenas com desenvolvimento típico, acrescidas de demandas extras decorrentes das exigências de cuidados com a criança DM. Essas demandas extras dependem do tipo de deficiência. Por exemplo, KRAUSS e SELTZER (1998), ao analisarem as conseqüências da deficiência mental ao longo do ciclo de vida familiar, mostraram que as mães de pessoas SD percebiam seus ambientes como mais coesos e 
menos conflituosos, relatando um nível mais elevado de satisfação com a rede social de apoio, bem como baixos níveis de estresse quando comparadas com outros grupos de mães de crianças DM. O temperamento e a adaptabilidade da criança SD foram vistos como aspectos facilitadores, embora o temperamento possa ser considerado também um fator de risco, principalmente quando favorece um tratamento diferencial dispensado pelos genitores aos filhos. Tal tratamento tem sido associado a resultados negativos no relacionamento entre irmãos, incluindo menos comportamento pró-social entre eles e maior competição e conflito (STONEMAN, 1998).

Os padrões de comportamento das mães podem favorecer similaridades nos perfis comportamentais das crianças com e sem DM. Segundo CRNIC (1990), as diferenças nos estilos de interação desenvolvidos entre os genitores de crianças DM e os de crianças com desenvolvimento típico estão no maior uso do controle e diretividade, menor responsividade, menos afeto positivo e reciprocidade, que caracterizam os padrões de interação de genitores de crianças DM. No entanto, as similaridades entre crianças com e sem DM parecem ser maiores que suas diferenças, particularmente quanto às categorias de interação mãe-criança SD (CIELINSKI et al., 1995). A maior diretividade das mães de crianças SD vem sendo associada por CIELINSKI et al., aos baixos níveis de desenvolvimento cognitivo de suas crianças e às sutis diferenças de comportamento, as quais devem ser mais exploradas em pesquisa futura.

Com relação ao pai, LAMB e BILLINGS (1997) ressaltam que, geralmente, eles reagem à deficiência de sua criança com a retirada do cuidado e das responsabilidades com a sua criação. $\mathrm{O}$ envolvimento limitado do pai da criança DM tem sido explicado: (a) pela sua inabilidade em lidar com uma criança com deficiência, (b) pelo desconforto em relação aos cuidados primários e (c) pela distribuição diferencial de papel na cultura, em que o cuidado dispensado à criança é considerado um domínio da mulher, enquanto os pais são vistos como responsáveis pela subsistência da família e como companheiros de brincadeiras da criança (MINNEs, 1998). Os dois últimos fatores refletem tanto o contexto específico de pais de crianças DM como sem DM.

No entanto, no estudo de PEREIRA-SILVA (2003), mães e pais de crianças com síndrome de Down iniciaram igualmente as interações com sua criança, enquanto em famílias de crianças com desenvolvimento típico a responsabilidade maior foi da mãe. O pai mostrou-se mais participativo nas famílias de crianças com síndrome de Down que nas famílias de crianças com desenvolvimento típico. Esses dados sugerem que é necessário investigar melhor as diferenças nos padrões de interação de famílias com e sem DM, considerando diferentes contextos culturais. Segundo LEWIS e DESSEN (1999), o envolvimento 
paterno está relacionado: (a) aos fatores psicológicos e sociais, (b) ao tipo de engajamento dos pais no sistema familiar e (c) às alterações nos modos de relação entre os diferentes subsistemas familiares, os quais sofrem influência do sistema social mais amplo.

A literatura tem procurado mostrar as diferenças e similaridades entre as famílias de crianças com e sem DM e os resultados encontrados por PEREIRA-SILVA (2003) confirmam a existência de padrões de interação mais similares que diferentes, particularmente entre famílias com e sem crianças com síndrome de Down. Neste estudo, as interações familiares caracterizaram-se pela 'Sincronia', 'Supervisão', 'Amistosidade' e 'Liderança'. Esse mesmo padrão foi encontrado em díades, tríades e tétrades, em ambos os tipos de família, tanto nas relações parentais quanto nas relações entre os irmãos. As atividades desenvolvidas pelos membros familiares e a criança foram, em sua maioria, em 'Grupo', havendo predominância da participação 'Conjunta', com a mãe sendo a maior responsável pela 'Supervisão' e 'Liderança' nas interações, em ambos os tipos de famílias.

Outro fator que merece ser destacado quanto ao processo de adaptação em ambos os tipos de família refere-se à dinâmica da relação do casal. Se, por um lado, há resultados indicando maiores taxas de conflito entre os casais em famílias de crianças DM que em famílias de crianças com desenvolvimento típico (SHAPIRO et al., 1998), por outro lado, há estudos que não encontraram diferenças quanto à satisfação marital entre esses dois tipos de família (BRISTOL et al., 1988; CARR, 1988). Para LAMB e BILLINGS (1997), essas diferenças podem ser atribuídas às variações quanto à idade, sexo e gravidade da deficiência.

Compreender como funcionam as famílias de crianças DM não é uma tarefa fácil. O avanço ocorrido na ciência do Desenvolvimento Humano, especialmente a partir de meados do século passado, e a ênfase atribuída às relações ocorridas dentro da família como um dos fatores preponderantes para a compreensão do desenvolvimento humano, particularmente nos primeiros anos de vida, têm suscitado reflexões e acarretado desafios metodológicos. Assim, finaliza-se este artigo chamando a atenção para duas questões fundamentais para a compreensão do desenvolvimento de crianças DM; a primeira, relacionada ao uso de uma abordagem metodológica apropriada para investigar os processos de adaptação familiar, e a segunda, sobre a influência genética e ambiental no desenvolvimento humano. 


\section{Considerações finais: um olhar para questões atuais de desenvolvimento humano}

Segundo LERNER, FISHER e WEINBERG (2000), a ciência do Desenvolvimento Humano envolve uma síntese de pesquisas que visam a promover o desenvolvimento saudável do indivíduo, ao longo do curso de vida, considerando as diferenças individuais, as mudanças intrapessoais e, também, os contextos familiar, social, cultural, ecológico e histórico. Para compreender o desenvolvimento de crianças DM é necessário, sobretudo, conhecer o seu contexto primário de desenvolvimento, isto é, a família. Tomando como base os pressupostos básicos da ciência do Desenvolvimento, que recurso metodológico poderíamos empregar em nossas pesquisas?

A abordagem bioecológica de BRONFENBRENNER $(1979 ; 1996 ; 1986 ; 1999)$ tem-se mostrado adequada para orientar pesquisas em desenvolvimento e, na área de deficiência mental, tem sido especialmente empregada por SHAPIRO et al. (1998). Neste modelo, a família é considerada um dos elementos chave para a compreensão do desenvolvimento humano, particularmente de crianças, constituindo o que BRONFENBRENNER denominou de microssistema. No microssistema família, por exemplo, as características da criança afetam diretamente os relacionamentos genitores-criança, assim como as características maternas e paternas. Crianças com problemas como desenvolvimento mais lento, falta de responsividade social, agressividade ou demandas de cuidados não usuais são associadas com crescente estresse, depressão, discórdia marital, pobreza no ajustamento materno e com uma diminuição da competência no papel paterno/materno (sHAPIRO et al., 1998). Essas características parecem ter grande influência no processo de adaptação das famílias, particularmente nas interações do dia-a-dia, conforme discutido neste artigo.

$\mathrm{O}$ mesossistema, que se refere às inter-relações "entre dois ou mais ambientes nos quais a pessoa em desenvolvimento participa ativamente" (BROFENBRENNER, 1979/1996, p. 21), pode contribuir para favorecer ou dificultar o funcionamento interno da família. Por exemplo, as relações positivas estabelecidas entre a família e a escola ou vizinhança são preditores consistentes da diminuição do estresse maternal e paternal, da sobrecarga e dos níveis de depressão, do bem-estar materno, do ajustamento psicológico e da competência no exercício das funções de pai e mãe (sHAPIRO et al., 1998). O exossistema consiste em sistemas que não se relacionam diretamente à pessoa em desenvolvimento, como o trabalho dos pais, mas que, de forma indireta, afetam essa pessoa. Por exemplo, o número de horas que o pai e/ou a mãe 
passa no trabalho e o estresse decorrente da sobrecarga de trabalho afetam a qualidade das interações pais-criança, em casa. Os sistemas sociais de apoio são importantes, na medida em que reduzem o estresse e a depressão, proporcionando uma melhoria no bem-estar e adaptação da família como um todo.

As influências da cultura e da etnia compõem os elementos característicos do macrossistema. Segundo SHAPIRO et al., (1998), é difícil especificar os efeitos que a etnia e a cultura têm sobre a adaptação das famílias de crianças DM, uma vez que estas questões raramente são investigadas. Esses autores relatam que mães de crianças com síndrome de Down da Escandinávia sentem menos depressão ou ansiedade e mostram sentimentos negativos logo após o nascimento de sua criança, enquanto mães afro-americanas sentem menos estresse que as caucasianas. As mães brasileiras também relataram diversas reações frente à deficiência do filho: choque, tristeza, revolta, resignação e culpa (BRUNHARA; PETEAN, 1999). Cada cultura parece apresentar reações diferentes ao nascimento da criança DM e pode ter um modo específico de lidar com a deficiência, que precisa ser melhor investigado.

Não se pode negar a importância do ambiente como um contexto fundamental para o desenvolvimento da criança. O modelo bioecológico de BROFENBRENNER (1999) contém premissas importantes que merecem destaque: (a) para o desenvolvimento ocorrer, a pessoa deve se engajar em uma atividade; (b) a efetividade do desenvolvimento depende da regularidade com que essa atividade ocorre durante períodos do tempo e de sua constância; (c) a atividade deve ter uma duração suficiente para tornar-se mais complexa; e (d) os processos não ocorrem somente na interação interpessoal, mas também na interação com objetos e símbolos (BROFENBRENNER, 1999; BROFENBRENNER; MORRIS, 1998). Trata-se, portanto, de um modelo interativo, na medida em que a interação entre as pessoas constitui uma de suas principais propriedades, sendo as demais decorrentes desta. Neste modelo, o desenvolvimento é “...uma função de forças emanando de múltiplos contextos e de relações entre esses contextos" (BROFENBRENNER; MORRIS, 1998, p. 1016). O indivíduo, com suas características pessoais, é parte ativa deste contexto, que influencia e é influenciado por ele.

Para PLOMIN (2000), uma das maiores mudanças na história da ciência comportamental é o reconhecimento da influência genética no comportamento dos indivíduos. Algumas descobertas recentes têm revolucionado a pesquisa, por exemplo, a de que a doença de Alzheimer está associada a um gene do cromossomo 19 e que a desordem do pânico também está associada à presença de um gene (PLOMIN; RUTTER, 1998). Nos estudos sobre inteligência, há uma evidência de hereditariedade em aproximadamente 50\% dos casos (PLOMIN; 
NEIDESHISER, apud SIMONOFF; BOLTON; RUTTER, 1998), indicando um efeito tanto genético quanto de influências não genéticas. Portanto, atualmente, os pesquisadores têm considerado tanto os efeitos do ambiente sobre o indivíduo como os genéticos, com alguns procurando descartar a importância dos efeitos genéticos e outros minimizando os efeitos do ambiente. Segundo BROFENBRENNER e CECI (1994), a trajetória de transformação dos genótipos em fenótipos recebe influência tanto de uma carga genética como dos efeitos dos processos proximais, que se referem às interações estabelecidas entre os indivíduos em suas atividades rotineiras. Portanto, é importante considerar a confluência de fatores atuando no desenvolvimento do indivíduo. Em relação à deficiência mental, é fundamental "...determinar como o entrelaçamento entre fatores genéticos e ambientais conduz à deficiência mental" (SIMONOFF et al., 1998, p. 42), se quisermos compreender o desenvolvimento de crianças DM.

Durante muito tempo a pesquisa tentou separar o interjogo "natureza e educação' (SIMONOFF et al., 1998), mas, atualmente, observa-se o ressurgimento do debate sobre as influências genéticas e ambientais, apontado como uma das principais tendências de pesquisa sobre desenvolvimento neste século que se inicia (LERNER et al., 2000; PLOMIN, 2000). Algumas questões são particularmente importantes e constituem grandes desafios para os pesquisadores do desenvolvimento, principalmente aquelas referentes aos papéis desempenhados pelos fatores genético e ambiental nas mudanças que ocorrem no decorrer do ciclo vital.

Acreditamos que a implementação de pesquisas que levem em consideração o desenvolvimento da criança DM inserida em um contexto familiar, também em desenvolvimento, procurando seguir as orientações do modelo bioecológico proposto por BRONFENBRENNER, contribuirá para o avanço do conhecimento sobre o desenvolvimento de crianças com deficiência mental e, conseqüentemente, de crianças com desenvolvimento típico. 


\section{REFERÊNCIAS}

ABRAMOVITCH, R. et al. The influence of Down's syndrome on sibling interaction. Journal of Child Psychology and Psychiatry and Allied Disciplines, v. 28, p. 865879, 1987.

ALI, M. R. et al. Parents of mentally retarded children: personality characteristics and psychological problems. Social Behavior and Personality, v. 22, p. 41-52, 1994.

BAGENHOLM, A.; GILLBERG, C. Psychosocial effects on sibling of children with autism and mental retardation: a population-based study. Journal of Mental Deficiency Research, v. 35, p. 291-307, 1991.

BOLSANELLO, M. A. Interação mãe-filho portador de deficiência: concepções e modo de atuação dos profissionais em estimulação precoce. São Paulo, 1998. 127 p. Tese (Doutorado em Psicologia) - USP.

BRISTOL, M. M.; GALLAGHER, J. J.; SCHOPLER, E. Mothers and fathers of young developmentally disabled and nondisabled boys: adaptation and spousal support. Developmental Psychology, v. 24, p. 441-451, 1988.

BRITO, A. M. W.; DESSEN, M. A. Crianças surdas e suas famílias: um panorama geral. Psicologia: Reflexão e Crítica, v. 12, p. 429-445, 1999.

BRODY, G. H.; STONEMAN, Z. A risk-amelioration model of sibling relationships: conceptual underpinnings and preliminary findings. In: BRODY, G. H. (Org.). Sibling relationships: their causes and consequences. Norwood: Ablex Publishing, 1996.

BRODY, G. H. et al. Observations of the role relations and behavior between older children with mental retardation and their younger siblings. American Journal on Mental Retardation, v. 95, p. 527-536, 1991.

BRONFENBRENNER, U. Ecology of the family as a context for human development: research perspectives. Developmental Psychology, v. 22, p. 723-742, 1986.

- A ecologia do desenvolvimento humano: experimentos naturais e planejados, (1979). Tradução: M. A. Veríssimo, Porto Alegre: Artes Médicas, 1996.

. Environments in developmental perspective: theoretical and operational models. In: FRIEDMAN, S. L.; WACHS, T. D. (Orgs.). Measuring environment across the life span: emerging methods and concepts. Washington D.C.: American Psychological Association, 1999.

; CECI, S. J. Nature-nurture reconceptualized in developmental perspective: a bioecological model. Psychological Review, v. 101, p. 568-586, 1994. 
; MORRIS, P. A. The ecology of developmental processes. In: DAMON, W.; LERNER, R. M. (Orgs.). Handbook of child psychology, v. 1 (Theoretical models of human development). New York: Wiley, 1998.

BRUNHARA, F.; PETEAN, E. B. L. Mães e filhos especiais: reações, sentimentos e explicações à deficiência da criança. Cadernos de Psicologia e Educação Paidéia, p. 31-40, 1999.

BYRNE, E. A.; CUNNINGHAM, C. The effects of mentally handicapped children on families: a conceptual review. Journal of Child Psychology and Psychiatry and Allied Disciplines, v. 5, p. 847-864, 1985.

CARR, J. Six weeks to twenty-one years old: a longitudinal study of children with Down's syndrome and their families. Journal of Child Psychology and Psychiatry and Allied Disciplines, v. 29, p. 407-431, 1988.

CASARIN, S. Aspectos psicológicos na síndrome de Down. In: SCHWARTZMAN, J.S. (Org.). Síndrome de Down. São Paulo: Mackenzie, 1999.

CIELINSKI, K. L. et al. Relations among sustained engagement during play, quality of play, and mother-child interaction in samples of children with Down syndrome and normally developing toddlers. Infant Behavior and Development, v. 18, p. 163-176, 1995.

COLnAGO, N. A. S. Pares mães-bebês síndrome de Down: estudo da estimulação e dos aspectos qualitativos da interação. São Carlos, 1991. Dissertação (Mestrado em Educação Especial) - UFSCAR.

CORTER, C. et al. Home observations of mothers and sibling dyads comprised of Down's syndrome and nonhandicapped children. Canadian Journal of Behavioural Science, v. 24, p. 1-13, 1992.

COWAN, C. P., COWAN, P. A. When partners become parents. New York: Wiley, 1992.

CRNIC, K. A. Families of children with Down syndrome: ecological contexts and characteristics. In: CICCHETTI, D.; BEEGHLY, M. (Orgs.). Children with Down syndrome: a developmental perspective. Cambridge: Cambridge University Press, 1990.

DESSEN, M. A. Desenvolvimento familiar: transição de um sistema triádico para poliádico. Temas em Psicologia, v. 3, p. 51-61, 1997.

DESSEN, M. A.; PEREIRA-SILVA, N. L. Deficiência mental e família: uma análise da produção científica. Cadernos de Psicologia e Educação Paidéia, v. 10, p. 12-23, 2000 .

DYSON, L. L. Fathers and mothers of school-age children with developmental disabilities: parental stress, family functioning, and social support. American Journal on Mental Retardation, v. 102, p. 267-279, 1997. 
EMERY, R. E.; FINCHAM, F. D.; CUMMINGS, E. M. Parenting in context: systemic thinking about parental conflict and its influence on children. Journal of Consulting and Clinical Psychology, v. 60, p. 909-912, 1992.

EREL, O.; BURMAN, B. Interelatedness of marital relations and parent-child relations: a meta-analytic review. Psychological Bulletin, v. 118, p. 108-132, 1995.

EREL, O.; MARGOLIN, G.; JOHN, R. S. Observed sibling interaction: links with the marital and the mother-child relationship. Developmental Psychology, v. 34, p. 288298, 1998.

FLOYD, F. J.; COSTIGAN, C. L.; PHILLIPPE, K. A. Developmental change and consistency in parental interactions with school-age children who have mental retardation. American Journal on Mental Retardation, v. 101, p. 579-594, 1997.

FLOYD, F. J.; ZMICH, D. E. Marriage and the parenting partnership: perceptions and interactions of parents with mentally retarded and typically developing children. Child Development, v. 62, p. 1434-1448, 1991.

FOX, L. et al. We can't expect other people to understand: family perspectives on problem behavior. Exceptional Children, v. 68, p. 437-450. Disponível em: < http:// proquest.umi.com/pqdweb>, Acesso em: 14 set. 2002.

GALLIMORE, R. et al. Family responses to children with early developmental delays II: accommodation intensity and activity in early and middle childhood. American Journal on Mental Retardation, v. 101, p. 215-232, 1996.

GANIBAN, J.; WAGNER, S.; CICCHETTI, D. Temperament and Down syndrome. In: CICCHETTI, D.; BEEGHLY, M. (Orgs.). Children with Down syndrome: a developmental perspective. Cambridge: Cambridge University Press, 1990.

GLIDDEN, L. M.; FLOYD, F. J. Disaggregating parental depression and family stress in assessing families of children with developmental disabilities: a multisample analysis. American Journal on Mental Retardation, v. 102, p. 250-266, 1997.

HODAPP, R. M.; BURACK, J. A.; ZIGLER, E. Developmental approaches to mental retardation: a short introduction. In: BURACK, J. A. et al. Handbook of mental retardation and development. Cambridge: Cambridge University Press, 1998.

HORNBY, G. Effects on fathers of children with Down syndrome. Journal of Child and Family Studies, v. 4, p. 239-255, 1995.

KERIG, P. K.; COWAN, P. A.; COWAN, C. P. Marital quality and gender differences in parent-child interaction. Developmental Psychology, v. 29, p. 931-939, 1993.

KNOTT, F.; LEWIS, C.; WILLIAMS, T. Sibling interaction of children with learning disabilities: a comparison of autism and Down's syndrome. Journal of Child Psychology and Psychiatry, v. 36, p. 965-976, 1995. 
KRAUSS, M. W.; SELTZER, M. M. Life course perspectives in mental retardation research: the case of family caregiving. In: BURACK, J. A. et al. Handbook of mental retardation and development. Cambridge: Cambridge University Press, 1998.

KREPPNER, $\mathrm{K}$. The child and the family: interdependence in developmental pathways. Psicologia: Teoria e Pesquisa, v. 16, p. 11-22, 2000.

LAMB, M. E.; BILLINGS, L. A. L. Fathers of children with special needs. In: LAMB, M. E. (Org.). The role of the father in child development. New York: Wiley, 1997.

LANDRY, S. H. et al. Effects of social context and mothers' requesting strategies on Down's syndrome children's social responsiveness. Developmental Psychology, v. 30, p. 293-302, 1994.

LEARY, P. M.; VERTH, F. The effect of a mentally retarded child on family functioning in a third world community. Early Child Development and Care, v. 109, p. 83-88, 1995.

LERNER, R. M.; FISHER, C. B.; WEINBERG, R. A. Applying developmental science in the $21^{\text {st }}$ century: international scholarship for our times. International Journal of Behavioral Development, v. 24, p. 24-29, 2000.

LEWIS, C.; DESSEN, M. A. O pai no contexto familiar. Psicologia: Teoria e Pesqui$s a$, v. 15 , p. 9-16, 1999.

LIEBERMAN, D.; PADAN-BELKIN, E.; HAREL, S. Maternal directiveness and infant compliance at one year of age: a comparison between mothers and their developmentally-delayed infants and mothers and their nondelayed infants. Journal of Child Psychology and Psychiatry and Allied Disciplines, v. 36, p. 1091-1096, 1995.

MARFO, K.; DEDRICK, C. F.; BARBOUR, N. Mother-child interactions and development of children with mental retardation. In: BURACK, J. A. et al. Handbook of mental retardation and development. Cambridge: Cambridge University Press, 1998.

MINNES, P. Mental retardation: The impact upon the family. In: BURACK, J. A. et al. Handbook of mental retardation and development. Cambridge: Cambridge University Press, 1998.

NEGRIN, N. S.; CRISTANTE, F. Resources and stress in parents with a mentally retarded child: a quantitative approach. In: CUSINATO, M. (Org.). Research on family: resources and needs across the world. Milão: LED - Edizioni Universitarie, 1996.

PEREIRA-SILVA, N. L. Crianças pré-escolares com síndrome de Down e suas interações familiares. Brasília, 2000, 126 p. Dissertação (Mestrado em Psicologia) Instituto de Psicologia, Universidade Nacional de Brasília.

. Famílias de crianças com e sem síndrome de Down: um estudo comparativo das relações familiares. Brasília, 2003. 263 p. Tese (Doutorado em Psicologia) - Instituto de Psicologia, Universidade Nacional de Brasília. 
; DESSEN, M. A. Deficiência mental e família: implicações para o desenvolvimento da criança. Psicologia: Teoria e Pesquisa, v. 17, p. 133-141, 2001.

. Síndrome de Down: etiologia, caracterização e impacto na família. Interação em Psicologia, v. 6, p. 167-176, 2002.

. Crianças pré-escolares com síndrome de Down e suas interações familiares. Psicologia: Reflexão e Crítica, v. 16, n. 3, 2003.

PLOMIN, R. Behavioural genetics in the 21st century. International Journal of Behavioral Development, v. 24, p. 30-34, 2000.

PLOMIN, R.; RUTTER, M. Child development, molecular genetics, and what to do with genes once they are found. Child Development, v. 69, p. 1223-1242, 1998.

$\mathrm{ROACH}, \mathrm{M}$. A. et al. The structure of mother-child play: young children with Down syndrome and typically developing children. Developmental Psychology, v. 34, p. 7787, 1998.

RODRIGUE, J. R.; MORGAN, S. B.; GEFFKEN, G. R. Psychosocial adaptation of fathers of children with autism, Down syndrome and normal development. Journal of Autism and Development Disorders, v. 22, p. 249-263, 1992.

ROEYERS, H.; MYCKE, K. Siblings of a child with autism, with mental retardation and with a normal development. Child: Care, Health and Development, v. 21, p. 305319, 1995.

SHAPIRO, J.; BLACHER, J.; LOPEZ, S. R. Maternal reactions to children with mental retardation. In: BURACK, J. A. et al. Handbook of mental retardation and development. Cambridge: Cambridge University Press, 1998.

SIGOLO, S. R. R. L. Diretividade materna e socialização de crianças com atraso de desenvolvimento. Cadernos de Psicologia e Educação Paidéia, v. 10, p. 47-54, 2000.

SIMONOFF, E.; BOLTON, P.; RUTTER, M. Genetic perspectives on mental retardation. In: BURACK, J. A. et al. Handbook of mental retardation and development. Cambridge: Cambridge University Press, 1998.

SLOPER, P. et al. Factors related to stress and satisfaction with life in families of children with Down's syndrome. Journal of Child Psychology and Psychiatry and Allied Disciplines, v. 32, p. 655-676, 1991.

STONEMAN, Z. Research on sibling of children with mental retardation: contributions of developmental theory and etiology. In: BURACK, J. A. et al. Handbook of mental retardation and development. Cambridge: Cambridge University Press, 1998.

STONEMAN, Z. et al. Ascribed role relations between children with mental retardation and their younger siblings. American Journal on Mental Retardation, v. 95, p. 537$550,1991$. 
TURNBULL, A. P.; RUEF, M. Family perspectives on problem behavior. Mental Retardation, v. 34, p. 280-293, 1996.

VAUGHN, B. E. et al. Quality of toddler-mother attachment in children with Down syndrome: limits to interpretation of strange situation behavior. Child Development, v. 65, p. 95-108, 1994.

Texto recebido em 15 jul. 2003 Texto aprovado em 24 nov. 2003 